\title{
PRO AND CONTRA CHANGES IN THE CONVENTIONAL VALUES OF PRECESSION
}

\author{
W. FRICKE \\ Astronomisches Rechen-Institut, Heidelberg, F.R.G.
}

\begin{abstract}
From an evalution of the arguments pro and contra changes in the precessional constants it is concluded that corrections should be made simultaneously with changes in the values of planetary masses at the time when the new fundamental reference system, the FK5, is introduced into the ephemerides. In this case the location and motion of the equinox would be corrected at the same time. An IAU decision on changes in the constants should be made in 1976. Since the completion of the FK5 may be expected around 1980, the introduction into the ephemerides appears practicable in 1984 .
\end{abstract}

\section{Introduction}

Whenever an improved fundamental reference frame shall be compiled and introduced into the ephemerides, a decision has to be made on the values of precession. Since 1960 the positions and proper motions of the fundamental stars given in the 'Fourth Fundamental Catalogue' (abbreviated FK4) represent the conventional reference system. This system requires a revision, and there is general agreement that work at the improvement must start as soon as possible on the basis of the observations that have become available since the completion of the FK4 and on observations of stars fainter than magnitude 7.5 which are not included in the FK4, but may be suitable for an extension of the fundamental system to a fainter magnitude limit. The goal of the programme that will result in a new fundamental catalogue, the FK5, is the improvement of the systematic and internal accuracy of the data of the FK4 and the inclusion of stars down to about magnitude 9.0 in the FK5. More details about the plans for the revision and extension of the FK4 and about observations relevant to this task have been reported by Fricke (1974) and by Gliese (1974). From the large observational material that has to be exploited it can hardly be expected that the FK 5 will be completed before 1980 .

The first international agreement on the use of precessional values was reached by the Conférence internationale des étoiles fondamentales de 1896 in Paris (ProcèsVerbaux, Gauthier-Villars, Paris, p. 54). This conference adopted Newcomb's (1898) values which are still in use at present. The compilers of the FK4 have applied Newcomb's precession on the basis of a recommendation made by the international colloquium on 'Constantes fondamentales de l'astronomie' held in Paris in 1950 (Publ. Centre Nat. Rech. Sci., Paris 1950, p. 129). After the completion of the FK4 no urgency has been seen for changes in precession. Consequently, in 1963, the $I A U$ Symp. 21 on 'The System of Astronomical Constants' held in Paris recommended that no change be made at that time, see Kovalevsky (1965), p. 322. It was, however, recognized that a reconsideration would be desirable at a time when the 'IAU System of Astronomical Constants' proposed by the Paris Symposium in 1963 and adopted 
by the IAU in 1964 (Trans. IAU XIIB, 593, 1966) should require amendments. The IAU Colloquium No. 9 on 'The IAU System of Astronomical Constants' held at Heidelberg in 1970 has considered this question and resolved "that any changes in the precessional constants and in the system of planetary masses be introduced into the national and international almanacs together, at a time that is closely linked with the introduction of the next fundamental star catalogue" (Celes. Mech. 4, 147, 1971). This resolution was endorsed by IAU Com. 4 in Brighton in 1970 (Trans. IAU XIIB, $81,1971)$, and a Working Group was set up to report, in time for consideration in 1973 , on the consequences of changes in the precessional constants and on the procedure for the introduction of new values at a later date. The term 'precessional constants' is used here in a wide sense to refer to a set of numerical expressions that define the mean positions of the equinox, equator and ecliptic at any epoch with respect to their positions at some standard epoch. In this connection the possible removal of the $E$-terms of aberration from the mean places of stars and the possible adoption of a new series for the nutation has to be considered.

The Working Group consisted of W. Fricke (chairman), T. Lederle (secretary), S. Aoki, S. V. M. Clube, J. Kovalevsky, J. H. Lieske, A. A. Nemiro, C. A. Lundquist, P. K. Seidelmann, S. Vasilevskis, and R. O. Vicente. The results of an exchange of views between the members of the Working Group are included in this report. Concerning the date for an IAU resolution on precessional constants, the Group has agreed that the decision should be made simultaneously with those on planetary masses, and that no final decision should be made at the IAU General Assembly in Sydney. From the view of the compilers of the FK5, the IAU should therefore decide on precession at one of its next General Assemblies, preferably in 1976. Hence, there is still an opportunity for considerations pro and contra changes.

\section{Strength of Evidence for New Precessional Values}

The following effects and quantities have to be discussed: (a) planetary precession, (b) lunisolar precession and a non-precessional motion of the equinox, (c) the obliquity and its secular changes, and (d) nutation.

\subsection{Planetary precession}

Two methods of determination are available. These are (1) the dynamical method based on planetary masses, and (2) the method of stellar kinematics which uses proper motions in a fundamental system and yields a simultaneous solution for lunisolar and planetary precession. The first method was applied by Newcomb, and it is even now the more powerful one. The second method is applicable, if the fundamental proper motions in right ascension are neither affected by any spurious motion of the equinox arising from errors in equinox determinations, nor by any component of real motions of stars parallel to the equator. At present, there is strong evidence for a fictitious motion of the equinox and still uncertainty about the 'real motions' of stars. Under these circumstances it is fortunate that the dynamical method yields results of an 
accuracy which may be satisfactory for a long time to come. Laubscher (1972) has determined the correction to Newcomb's value of the first order term in planetary precession on the basis of the planetary masses given by Klepczynski et al. (1971), and he has estimated its uncertainty by taking into account the ranges of the values for various planetary masses given by Kovalevsky (1971). Laubscher's result is

$$
\Delta \lambda=-0.03 \pm 0.01 \text { per century, }
$$

which means, (1) that the improvements made in the determination of planetary masses since the time of Newcomb have been of small effect on planetary precession, and (2) that future improvements will hardly change the correction significantly. I understand that an investigation made by $\mathrm{Dr}$ J. D. Mulholland is in agreement with this result.

\subsection{LUNISOLAR PRECESSION AND A FICTITIOUS MOTION OF THE EQUINOX}

Appreciable corrections to Newcomb's lunisolar precession have been derived by many authors since about 1910. Although it is not intended to review here the numerous determinations, it should be mentioned that Boss (1910) was the first to derive values on the basis of a proper motion system that was superior to Newcomb's system. Boss had no knowledge of the effects of galactic rotation at that time, but his results were later on found to be in the right direction. He also confirmed what Newcomb already had suspected, namely, that the equinox determined from observations of the Sun and planets prior to 1870 must be considerably in error.

Later on, when improved proper motion systems had become available - the GC, FK3, and the N30 -, and the effects of galactic rotation had been introduced into the solutions as additional unknowns, corrections $\Delta n$ to Newcomb's value of precession in declination were derived in the range

$$
+0 " 30 \lesssim \Delta n \lesssim+0.60 \text { per century. }
$$

A list of such determinations has been given by Böhme and Fricke (1965). Among these are some values which were not derived for the purpose of contributing to a better knowledge of astronomical constants but resulted as a by-product of investigations on stellar motions. Some other determinations in the list are affected by traceable errors. Two investigations deserve mentioning in which the authors made attempts to estimate the most probable values on the basis of older determinations. These are the investigations by Gordon (1952) and by Morgan and Oort (1951). Gordon applied a fairly primitive averaging procedure, while Morgan and Oort used a much more refined one, but made a traceable error in the reduction of results from one reference system to the other. More details on the comparison of precessional corrections derived on the basis of different systems were given by Fricke (1967a, b). Gordon's result of averaging is

$$
\begin{aligned}
\Delta p_{1} & =+1^{\prime \prime} .09 \pm 0^{\prime \prime} .03 \text { per century, (corresponding to } \Delta n=+0^{\prime \prime} .44 \text { ) } \\
\Delta \lambda+\Delta e & =+1^{\prime \prime} .24 \pm 0.03 \text { per century. }
\end{aligned}
$$


( $\Delta p_{1}$, correction to lunisolar precession; $\Delta \lambda$, correction to planetary precession; $\Delta e$, correction to all proper motions in right ascension due to a fictitious motion of the equinox.)

The completion of the FK4 in 1963 suggested new investigations with the aim to find the precessional corrections in the FK4 system, and, by the application of the same stars, in the older systems, the GC, FK3, and N30. In order to avoid errors due to large proper motions, stars had to be selected in FK4 and FK4 Sup with distances larger than about $100 \mathrm{pc}$. The result was unexpected and surprising. On the basis of FK4 proper motions Fricke (1967b) found

$$
\begin{aligned}
\Delta n & =+0.44 \pm 0.06 \text { per century }, \\
\Delta \lambda+\Delta e & =+1.20 \pm 0.15 \text { per century. }
\end{aligned}
$$

The errors are standard deviations. The correction $\Delta n$ turned out to have the same value, within 0.01 per century, in the systems FK3, N30, and FK4, while $\Delta \lambda+\Delta e$ is slightly but not significantly different in the three systems.

In an effort to ascertain the reason for the deviation of Newcomb's results from those derived on the basis of FK3, N30, and FK4, Fricke (1971a) has made a rediscussion of Newcomb's determination. For a summary of the results reference is made to Fricke (1971b). It has been found that the value $\Delta n$ given above is almost entirely the consequence of deficiencies of Newcomb's proper motion system in declination, and that the value $\Delta \lambda+\Delta e$ originates from deficiencies in Newcomb's determination of the equinox. The widespread suspicion that Newcomb's results were mainly affected by the neglect of galactic rotation or by an erroneous analysis of the proper motions has not been confirmed. On the contrary, Newcomb's analysis can be considered as an example of great mastery, and Newcomb cannot be blamed for the imperfections in the proper motion system available to him. The equality of the values of lunisolar precession in the systems FK3, N30, and FK4 demonstrates that in the 20 th century the fundamental declination system has become an almost stable one. This has been confirmed by observations made within the past 20 years, which will hardly change the FK4 system in declination significantly. Some serious deficiencies in the FK4 which have recently become apparent lie in the right ascension system in the southern sky; but their nature is such that they cannot affect determinations of precession. These facts may be considered as strong arguments in favour of changes in the precessional constants.

The explanation of the large fictitious motion of the equinox

$$
\Delta e=+1 \text {.'23 per century, }
$$

where $\Delta \lambda=-0^{\prime \prime} .03$ is taken into account, must come from the observations of the Sun and other bodies of the planetary system. Their analysis is being carried out at Heidelberg. This is one of the most urgent tasks within the work at the improvement of the fundamental right ascension system. There are clear indications that $\Delta e$ is the consequence of systematic errors of observations. One of the more recent investigations confirming these indications has been carried out by Martin and van Flandern 
(1970) and van Flandern (1971) who have determined the motion of the FK4 equinox on the basis of lunar occultation observations. They found $\Delta e=+1^{\prime \prime} .36 \pm 0$ ".06 per century which is in good agreement with the results from proper motions. The observations of the Sun, of planets and of lunar occultations may also help to decide whether part of the equinox motion arises from some systematic motions of the stars as suggested by Clube (1972) and by Vasilevskis and McNamara (1973). Undoubtedly this possibility cannot be entirely excluded on the basis of fundamental proper motions alone.

There are other methods which, in principle, allow the determination of precessional constants. None of these has so far given satisfactory results or any result that could cast doubt on the values derived from fundamental proper motions. At the Jet Propulsion Laboratory it is planned to apply the dynamical method based on planetary observations. Dr Lieske has communicated that, at JPL, the optical planetary data from the U.S. Naval Observatory have been reduced to the FK4 for the purpose of exploring the possibilities of solving for precessional corrections. Since the radar data are insensitive to the correction to general precession, it is hoped that the precessional correction and $\Delta e$ can be separated.

Another method consists in the comparison of proper motions measured with respect to galaxies with those in the fundamental system. This method which has been applied by Fatchikhin (1970) and by Vasilevskis and Klemola (1971) has given some encouraging results. Apart from still unexplained discrepancies between the right ascension systems measured at the Lick Observatory and at Pulkovo, both determinations have yielded corrections $\Delta n$ in the direction of the value on the FK4 (Fatchikhin's value is, in fact, identical with the FK4 value). Reference is made to the brief review of these results by Fricke (1972). Final conclusions from proper motions with respect to galaxies will have to wait for the completion of the programmes at both observatories and for measurements in the southern sky, and thus cannot be expected soon.

For the future, there remains the possibility of determining precession from absolute radio astrometric measurements of compact extragalactic radio sources by means of radio interferometers. A study of the potentialities of the method has been made by Walter (1974), but so far no plans for observing programmes have become known.

In summarizing this review of the strength of evidence for new precessional values it must be concluded that, if a decision for changes in precession is made in 1976, a value for lunisolar precession would have to be adopted from the determinations based on proper motions in the systems FK3, N30, and FK4, and the value for planetary precession would have to be adopted from a determination based on planetary masses. The elimination of a non-precessional motion of the equinox has to result from the discussion of observations of the Sun, Moon, and planets, and it must be done in the FK5.

Arguments against a change in the precessional values are, first, the hypothetical nature of the method of determination by means of proper motions, and, second, that erroneous values of precession do not result in erroneous ephemerides, if the ephemerides are computed rigorously. Letters from Dr Clube and Dr Vasilevskis have re. 
minded me that these arguments should not be forgotten. In fact, I have used these arguments myself in 1964, (see Fricke, 1966), and given full consideration to these questions since that time. The first argument is correct, since precession cannot be determined without any hypothesis. In using fundamental proper motions the question as to the origin of the inherent rotation has to be answered. The rotation observed in the proper motions is the cumulative effect of various physically comprehensible and some spurious rotations. The hypothesis is made that the rotation arises from incorrect precession, galactic rotation and a non-precessional motion of the equinox caused by observational errors. From my own investigations I would not exclude that additional rotational effects may be present, but these effects are of the second order except when unsuitable observational material is applied. Concerning the second argument $I$ have convinced myself that in the past incorrect precessional values have done harm in the computation of ephemerides and particularly in the reduction of observations by observers. The fatality of incorrect constants is the unforeseen confusion they produce and the great effort required for tracing their effects in the various applications.

\subsection{Obliquity AND ITS SECUlar CHANGe}

For determining the value of the obliquity a rediscussion of the results obtained from the observations of the sun and the planets is necessary. The secular change of the obliquity has to follow, in conformity with the procedure applied by Newcomb, from planetary precession. Should the observations indicate a significant excess secular change, as it was taken for granted by Aoki (1967), then the origin of the effect would have to be found. As a preliminary result of own investigations I can report that the individual values of $\Delta \varepsilon$ from observations of the Sun and planets show indeed a decrease from about 1780 to about 1900. The values of $\Delta \varepsilon$ derived from observations after 1900, however, do not deviate sensibly from zero and do not show a significant secular change. There are similarities between the problems existing in the determinations of the equinox and the obliquity. In both, the older observations give rise to difficulties which cannot be easily solved. It may be mentioned that absolute observations carried out before about 1890 show not only a large scatter but also clear indications of neglected instrumental errors, so that the right ascension system of the FK4 had to be based on observations from about 1900 onwards exclusively, and that observations before 1900 entered the proper motion system of the FK4 with very small weight. It is to be expected that the FK5 will not bring a revival of the observations of the 19th century. This means that we will have to determine the equinox and the obliquity from observations made during the 20th century, and there is every indication that no significant variation of the obliquity in excess of the theoretical one is present.

\subsection{NUTATION}

Determinations of the principal nutation in obliquity, the constant of nutation, have been reviewed by Vicente (1971). Observations after 1900 have led to a value 
which is smaller than Newcomb's $(N=9.21)$ by about 0 " 01 with a mean error of a few units in the third decimal place. The smallness of the correction, the incomplete analysis of available observations, and the continuation of theoretical studies suggest that, at present, no change in the conventional value should be considered. I have noticed that Prof. Vicente supports this opinion, and no other suggestion has become known to me. The series developments of the nutation which have been used in the past in a consistent way have turned out to be of great advantage.

\section{Changes in the Procedures for Computing Precise Places}

When changes in the ephemerides are made, various suggestions for changes in the procedures for computing precise places may be taken into account. It is not intended to discuss here all such changes which may be considered. But, without going into details, two proposals made by Atkinson $(1951,1973)$ shall be mentioned here (cited from Dr Atkinson's letters).

(1) It would be helpful in all observational work, if all Apparent Right Ascensions were computed for the mean equinox of date, and not for the true equinox. At present, whether they are tabulated in the Ephemeris (or in the APFS) or are computed in the standard way by an observer, they must all have the Equation of Equinoxes subtracted from them, before they can be compared with transit-times read on a modern clock.

(2) It would benefit all observers of precise places, if the nutation were computed for the Earth's Pole of Figure and not for its Instantaneous Pole of Rotation.

\section{Form of Changes in the Precessional Constants}

New precessional constants can be introduced in a number of ways, and a particular method has to be adopted. One of the possibilities is to change explicitly the values of the general precession in longitude per tropical century and of the obliquity of the ecliptic for 1900.0 - these are the quantities included in the current IAU System of Astronomical Constants -, and the corresponding secular variations. Dr Seidelmann has drawn my attention to the desirability of a new definition of general precession that should include the relativistic effect and that would be clearly stated at the time when a decision on the constants is made. Work on the form of changes and definitions is ungratifying before a decision on the desirability of changes of the constants has been made, in particular, since the amount of work involved is considerable.

The consequences of changes have to be made known to those who prepare ephemerides and to all users, and formulae can easily be given for the reduction from the old to a new system.

Since the completion of the new fundamental reference system, the FK5, can hardly be expected before 1980, a realistic date for the simultaneous introduction of all changes into the ephemerides appears to be 1984 . 


\section{References}

Aoki, S.: 1967, Publ. Astron. Soc. Japan 19, 585.

Atkinson, R. d'E.: 1951, Monthly Notices Roy. Astron. Soc. 111, 619.

Atkinson, R. d'E.: 1973, Astron. J. 78, 147.

Böhme, S. and Fricke, W.: 1965, in J. Kovalevsky (ed.), 'The System of Astronomical Constants', IAU Symp. 21 B Bull Astron. 25, 269.

Boss, L.: 1910, Astron. J. 26, 111.

Clube, S. V. M.: 1972, Monthly Notices Roy. Astron. Soc. 159, 289.

Fatchikhin, N. V.: 1970, Astron. Zh. 47, 619 三Soviet Astron. 14, 495.

Fricke, W.: 1966, Trans. IAU XIIB, p. 604.

Fricke, W.: 1967a, Astron. J. 72, 642.

Fricke, W.: 1967b, Astron. J. 72, 1368.

Fricke, W.: 1971a, Astron. Astrophys. 13, 298.

Fricke, W.: 1971b, Celes. Mech. 4, 150.

Fricke, W.: 1972, Ann. Rev. Astron. Astrophys. 10, 101.

Fricke, W.: 1974, in W. Gliese, C. A. Murray, and R. H. Tucker (eds.), 'New Problems in Astrometry', IAU Symp. 61, 23.

Gliese, W.: 1974, in W. Gliese, C. A. Murray, and R. H. Tucker (eds.), 'New Problems in Astrometry, IAU Symp. 61, 31.

Gordon, J. E.: 1952, Izv. Glav. Astron. Obs. Pulkovo 19, No. 148, 72.

Klepczynski, W. J., Seidelmann, P. K., and Duncombe, R. L.: 1971, Celes. Mech. 4, 253.

Kovalevsky, J. (ed.): 1965,. 'The System of Astronomical Constants', IAU Symp. 21, 322.

Kovalevsky, J.: 1971, Celes. Mech. 4, 213.

Laubscher, R. E.: 1972, Astron. Astrophys. 20, 407.

Morgan, H. R. and Oort, J. H.: 1951, Bull. Astron. Inst. Neth. 11, 379.

Newcomb, S.: 1898, Astron. Papers Washington, 8, Part 1.

Van Flandern, T. C.: 1971, Celes. Mech. 4, 182.

Vasilevskis, S. and Klemola, A. R.: 1971, Astron. J. 76, 508.

Vasilevskis, S. and McNamara, B. J.: 1973, Astron. J. 78, 639.

Vicente, R. O.: 1971, Celes. Mech. 4, 186.

Walter, H. G.: 1974, in W. Gliese, C. A. Murray, and R. H. Tucker (eds.), 'New Problems in Astrometry', IAU Symp. 61, 131.

\section{DISCUSSION}

Mulholland: The argument against change that an incorrect value of the precession need not affect the accuracy of ephemerides was true in 1964, but is no longer. We now have observations in the third coordinate, distance, for three planets and the Moon. In addition, independent means exist for determination of masses. The effect of these new tools is that an error in precession cannot so readily be absorbed into erroneous mean motions.

Murray: I am not in favour of a change in the adopted constant of precession. Any value derived from analyses of proper motions depends on hypotheses about the stellar velocity pattern, and can never be truly fundamental.

A so-called fundamental system, such as for example FK5, must be regarded as a conventional system. It does not matter if it has a small net rotation relation to the inertial system.

Vicente: About the needs of a certain IAU commission to have a change of the system of astronomical constants only in 20 years' time, I should like to emphasize that we have to consider the problem of changing the constants not only in relation to the needs of one of our commissions but the needs in the whole field of astronomy.

Eichhorn: Two years ago, I would have welcomed a change of the constant of luni-solar precession to the best available value. This is not true anymore, and there are two reasons for this.

(1) During the last two years, the advent of radio-astrometry, and especially its potentialities, allows one to expect that these measurements will make an important contribution toward a more accurate value of the constant of precession. It may be indicated to wait for 20 years and incorporate these data.

(2) We have been reminded that stellar kinematics is more complicated than the Oort model, and 
that its description requires more parameters than this model provides. Inspite of all precautions, this fact may influence the best possible values of $n$ and $k$ which can be obtained from the material available even at this time.

Lastly, it is well known that a catalogue system is different from an inertial system. If the FK5 were too good an approximation to an inertial system, this fact might be overlooked by some investigators who should not overlook it.

For these reasons, it seems that the next 5 or 10 years might not be the most auspicious time for a change of the constant of luni-solar precession.

Fricke: The argument that more accurate determinations of luni-solar precession may become available in the near future can be used at any time. With our present knowledge, the accuracy of the value can be increased by one order of magnitude. Second order effects may be detected by radio astrometric methods or on the basis of improved stellar kinematics. The argument that the adoption of corrections to Newcomb's precession would lead to an FK5, which is a too good approximation to an inertial system is one which $I$ would use in favour of changes.

Eichhorn: The contribution of radio-astronomers in the next 20 years will be spectacular as more and more definite material is being accumulated, so we can wait as the difference between the FK4 system and an inertial system is known.

Kovalevsky: All the consequences of having a wrong value of the constant of precession on a very precise dynamical theory have not been completely studied and I believe that it is safer to have a newer and better value for this constant.

Tucker: Dynamical people should do what they like when necessary.

Vasilevskis: Do not forget the need of stellar astronomy and I concur with the remark by Dr Murray.

Vicente: When I mentioned that we cannot consider only the objectives of stellar astronomy, I did not mean they are not important objectives, but the working group has to take into account the needs and objectives of all IAU commissions and, therefore, I consider the date 1980 as a convenient one for a change of the system of astronomical constants. 